\title{
MR imaging of uncommon complications of transforaminal
}

\author{
Tan AP* and Quek ST \\ Department of Diagnostic Radiology, National University Hospital, Singapore
}

\begin{abstract}
Transforaminal epidural steroid injection (TFESI) is a common procedure in the management of spinal pain, as part of a conservative care treatment plan. It has emerged as an alternative to interlaminar and caudal epidural steroid injections in the past decade due to its efficacy in the management of spinal pain. Transforaminal epidural steroid injection (TFESI) is an important therapeutic and diagnostic procedure in the armamentarium of physicians specializing in the management of spinal pain. The putative reason for the effectiveness of transforaminal injection is due to the ability of the interventionist to place medications in the ventral aspect of the epidural space, the area most likely to be affected by disk degenerative disease. As the world heads into an era of dramatic demographic transformation where aging individuals encompass a larger proportion of the population, spinal degenerative disorders would be expected to be on the rise. Therefore, it is very important that radiologists and clinicians managing patients with spinal pain are able to recognize possible complications of transforaminal epidural steroid injection (TFESI) on magnetic resonance imaging (MRI), the modality of choice in detecting uncommon yet devastating complications such as cord infarct, brain infarct, subdural and epidural haematoma, arachnoiditis and meningitis. Familiarity with the appearances of these devastating and catastrophic complications on magnetic resonance imaging would not only hasten the diagnosis but also improve patient's overall outcome. The objective of this pictorial review is to unravel the uncommon yet devastating complications of transforaminal epidural steroid injection (TFESI) on magnetic resonance imaging (MRI).
\end{abstract}

\section{Introduction}

Catastrophic complications such as cord and brain infarct, subdural hematoma, epidural abscess, meningitis and arachnoiditis are uncommon [1], with an incidence of approximately $0.9 \%$ [2]. Spinal cord or brainstem infarction following TFESI is one of the rarest yet most devastating complications [2]. MR imaging has become the primary imaging modality in patients with neurological deficit or pain post procedure and is highly sensitive in demonstrating these entities. Hence, it is important that radiologists are familiar with the appearance of these complications on magnetic resonance imaging.

\section{Relevant anatomy}

Intervertebral foramina are tear-drop shaped openings (Figure 1) which contain the spinal nerve root, dorsal root ganglion, the spinal artery and communicating veins between the internal and external plexuses.

At typical cervical levels, the intervertebral foramina are oriented obliquely, anteriorly and laterally. The roof and floor of the intervertebral foramen are formed by the pedicles of contiguous vertebrae. Its posterolateral wall is formed by the superior and inferior articular processes while its anteromedial wall is formed by the caudad portion of the upper vertebral body, the uncinate process of the lower vertebral body and the posterolateral corner of the intervertebral disc. Immediately lateral to the external opening of the foramen is the vertebral artery which traverses anterior to the articular pillars of the zygapophyseal joint. Radicular arteries arise from the vertebral artery and the ascending cervical artery and accompany the spinal nerve and its roots to the spinal cord.

At lumbar levels, intervertebral foramina are oriented laterally. The roof and floor are formed by the pedicles of consecutive vertebrae. The

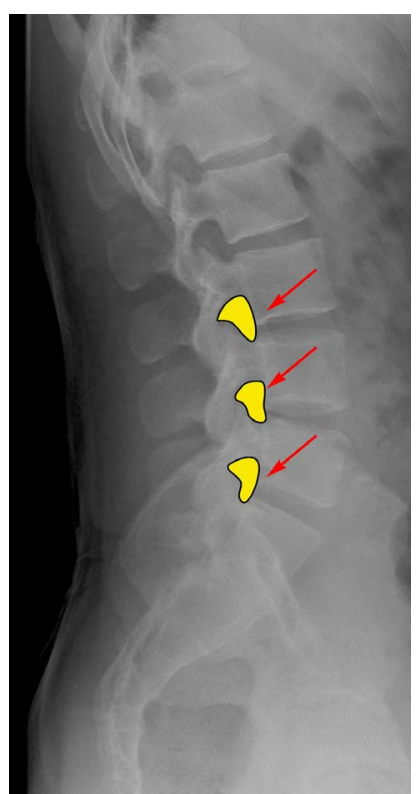

Figure 1. Inverted teardrop configuration of the neural foramina (red arrows) in the lumbar spine

Correspondence to: Ai Peng Tan, Department of Diagnostic Radiology, National University Hospital, Lower Kent Ridge Road, Singapore; E-mail: ai_peng_tan@nuhs.edu.sg

Key words: cord infarct, brain infarct, subdural hematoma, transforaminal epidural steroid injection, meningitis, arachnoiditis

Received: September 20, 2017; Accepted: October 17, 2017; Published: October 20,2017 
posterolateral wall is formed largely by the superior articular process of the lower vertebra and in part by the inferior articular process of the upper vertebra. The anteromedial wall is formed by the caudad end of the upper vertebral body and the posterolateral corner of the intervertebral disc. In the cephalad half of the foramen, the dorsal root ganglion lies just deep to the pedicle of the cephalad vertebra. Radicular arteries which arise from the abdominal aorta accompany the spinal nerve and its roots to the spinal cord.

\section{The procedure}

Transforaminal epidural steroid injection (TFESI) is a procedure designed to deliver an aliquot of a corticosteroid preparation to the immediate vicinity of a spinal nerve root via the intervertebral foramen in which the target nerve lies. This procedure provides better delivery of medication to the site of radiculopathy and increased spread into the ventral epidural space. The spinal needle is advanced into the neural foramen under direct fluoroscopic or CT guidance

The techniques of transforaminal epidural steroid injection differ slightly in the cervical and lumbar spine.

In the cervical spine, the needle is passed into the neck through a skin puncture which overlies the posterior half of the target foramen and is directed along the axis of the target foramen. Once the needle reached the superior articular process, the appropriate depth is reached. The tip of the needle is finally adjusted so it lies opposite the parasagittal midline of the articular pillars. Beyond this depth, there will be increased risk of dural sleeve or thecal sac puncture. The needle must stay in contact with the posterior wall to avoid vertebral artery injury (Figure 2).

In the lumbar spine, transforaminal epidural steroid injection is typically performed with the patient in prone position. Through a skin puncture overlying the superior portion of the target foramen, just caudal to the pars interarticularis, a needle is passed into the back (Figure 3). The tip of the needle should always lie over the posterior aspect of the intervertebral foramen. Once the needle reached the par interarticularis, the needle is advanced slowly till the target nerve is correctly outlined.

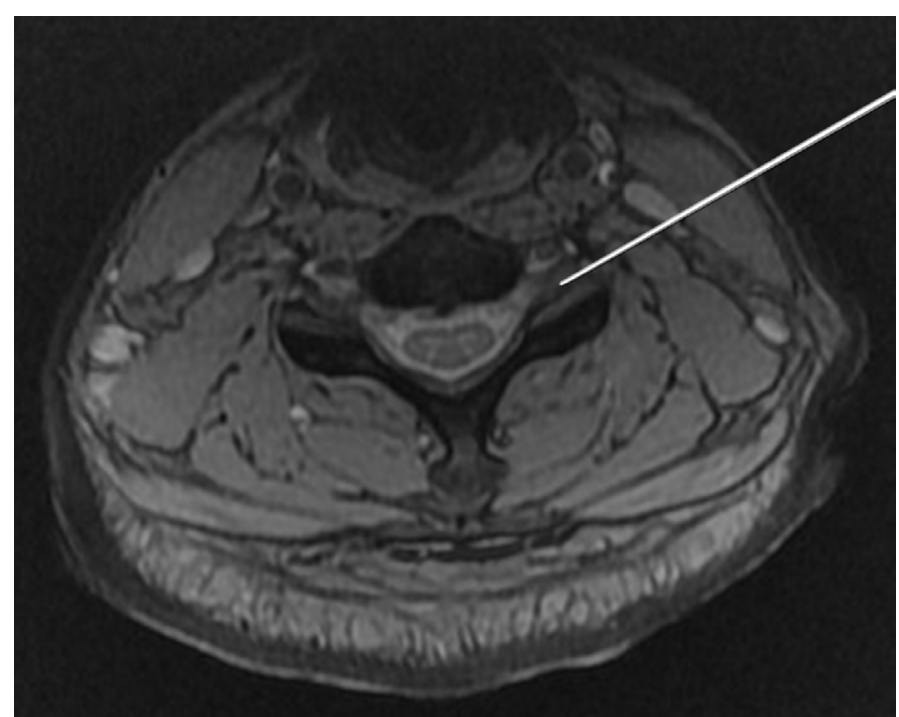

Figure 2. In the cervical spine, the needle is passed into the neck through a skin puncture and is directed along the axis of the target foramen. The needle must stay in contact with the posterior wall to avoid vertebral artery injury

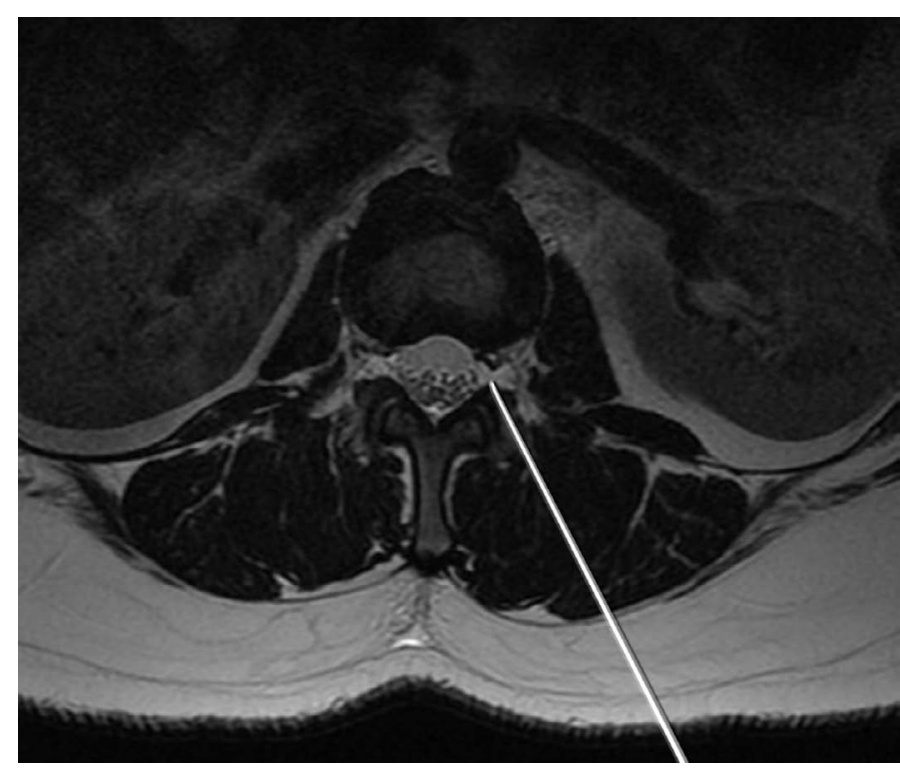

Figure 3. In the lumbar spine, transforaminal epidural steroid injection is typically performed with the patient in prone position. Through a skin puncture overlying the superior portion of the target foramen, just caudal to the pars interarticularis, a needle is passed into the back

Contrast material is injected to confirm epidural flow and to avoid intravascular or intradural and soft tissue infiltration. Intraarterial injection manifests by extremely rapid clearance of the injected contrast material. In a vertebral artery, the contrast material streaks in a cephalad manner. In a radicular artery, contrast blushes briefly in a transverse manner, medially towards the spinal cord. Rapid dilution of contrast material implies subarachnoid spread, which may occur if the needle punctures the thecal sac when there is lateral dilation of the dural root sleeve into the intervertebral foramen.

\section{Cord infarct}

Cord ischemia could result from vascular injury with thrombosis, embolism or from occlusion secondary to intra-arterial injection of steroid suspension [3]. The mechanism of injury could also be secondary to arterial spasm or the development of an intimal flap. Both of these mechanisms could lead to stasis of flow, clot formation, and hypoperfusion.

Patients often present with abrupt onset and rapid progression of weakness or loss of sensation. Patients with anterior spinal syndrome develop paralysis, pain and temperature sensation loss, bladder and bowel dysfunction while those with posterior spinal cord infarction present with loss of vibration sense and proprioception. In addition, pain is frequent and a disabling feature of cord infarct. It is also not uncommon for patients to have permanent disabling sequelae. Initial severity of neurological deficit is the best predictor of outcome.

MRI is the best imaging modality for diagnosis of cord infarct. On T1-weighted images (Figure 4b), the cord is slightly expanded and demonstrates low signal intensity due to the presence of oedema. In some patients $\mathrm{T} 1$ hyperintense foci may be seen within the infarcted cord due to hemorrhagic conversion. These will show blooming on gradient recalled echo imaging. On T2-weighted images (Figure 4a), high signal is seen within the cord, which may involve the central gray matter or the entire cross-sectional area of the cord [2]. On diffusionweighted imaging, the infarcted cord would show high signal with corresponding low signal on ADC map [2]. 


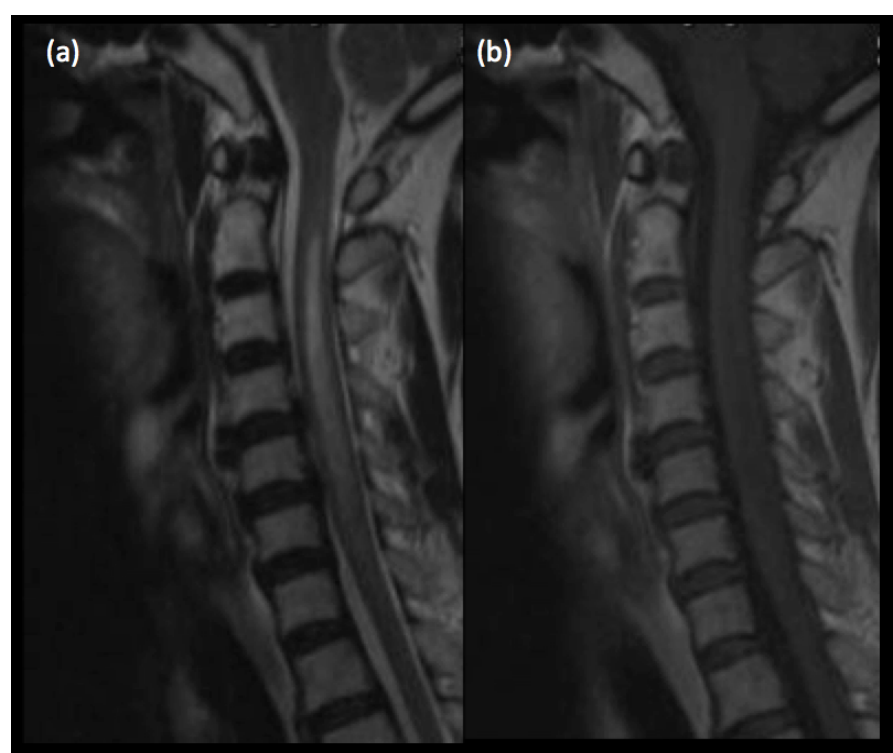

Figure 4. (a)Sagittal T2-weighted MRI shows hyperintense signal within the cervical cord (b)Corresponding low signal within the cervical cord on T1-weighted MRI is consistent with cord infarct

Patients with cord infarct are treated with steroids and anticoagulation. Supportive care and rehabilitative physical therapy are also of equal importance.

\section{Brain infarct}

Air embolism can cause infarctions in the posterior circulation. Other potential mechanisms of infarction include arterial perforation causing thrombosis, needle-induced vasospasm, intra-arterial corticosteroid injection and arterial dissection, similar to mechanisms of spinal infarction [4]. Brain infarcts invariably involve the cerebellum, brainstem (Figures 5, 6a and 6b) or posterior cerebral artery territory. Involvement of the optic radiation and lateral geniculate body would result in hemianopsia while involvement of the thalamic nuclei would cause hemisensory loss. Other possible presenting symptoms include vertigo, ataxia, syncope and dysarthria.

MR imaging is excellent in depicting brain infarcts, diffusionweighted imaging being the most sensitive in the acute settings. Infarcted brain parenchyma would be hyperintense of T2-weighted images and hypointense on T1-weighted images, due to the presence of cytotoxic oedema.

\section{Subdural hematoma}

Vascular injury is an inherent risk to all injection procedures, including transforaminal epidural steroid injection [5]. Potential complications associated with this are bleeding and the formation of hematomas [5].

Subdural hematomas can lead to compression of the spinal cord and nerve roots, causing irreversible damage [6]. Surgical evacuation may be necessary to alleviate compression of spinal cord or nerve roots, preferably within 24 hours to minimize neurological deficit. Without significant neurologic compromise, conservative management with follow up imaging may be considered.

Findings on magnetic resonance imaging would include a subdural fluid collection, the MR signal characteristics of which depend on the age of the hematoma (Figures 7a, 7b, 7c). Acute hematoma which contains deoxyhemoglobin is iso- to hypointense on T1-weighted images and hypointense on T2-weighted images. Intracellular methemoglobin within early subacute hematoma is T1 hyperintense and T2 hypointense while extracellular methemoglobin within late subacute hematoma is hyperintense on both T1- and T2-weighted images.

\section{Arachnoiditis}

Inflammation of the arachnoid, one of the membranes that enclose the spinal cord is known as arachnoiditis. Arachnoiditis is more common following multiple intrathecal injections. Hence, it is important to prevent puncturing the thecal sac during transforaminal epidural steroid injection. Patients with arachnoiditis may present with radicular or non radicular pain, motor or sensory alteration, gait disorder, bladder or bowel dysfunction.

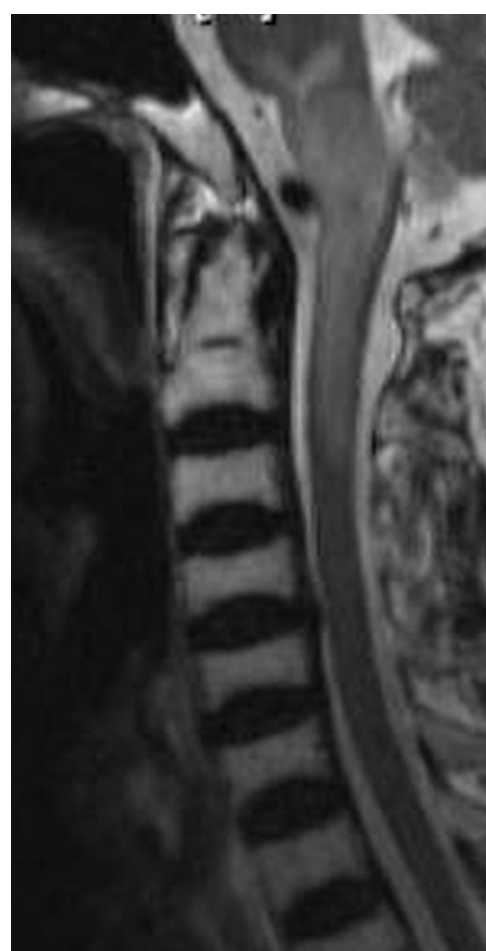

Figure 5. Sagittal T2W MRI shows increased signal within the brainstem and upper cervical cord in a patient with cord and brainstem infarct post TFESI

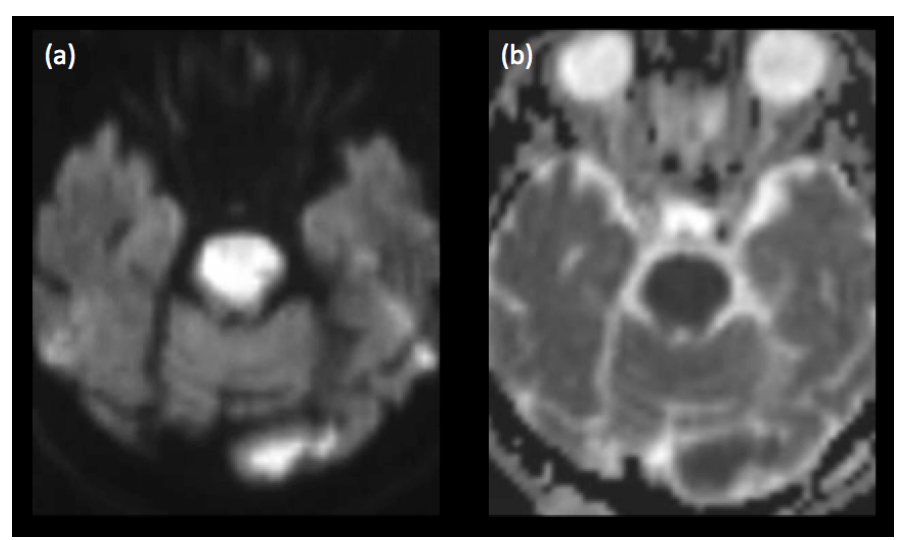

Figure 6. (a)Diffusion-weighted imaging and (b)corresponding ADC map reveal an acute pontine infarct in a patient with sudden onset of weakness post transforaminal steroid injection for symptomatic treatment of cervical spondylosis 
This is a rare complication following transforaminal epidural steroid injection and is characterized by clumping of the caudaequina nerve roots (Figure 8c), intrathecal fluid collection, enhancing nerve roots (Figures $8 \mathrm{a}$ and $8 \mathrm{~b}$ ), pial and dural enhancement. "Empty sac" sign in patients with arachnoiditis is due to nerve roots adhering to the periphery of the dural sac.

\section{Meningitis}

Infection is rare, occurring in approximately $1-2 \%$ of spinal injections [7]. Severe infections are noted to be even rarer with an incidence of $0.1-0.01 \%$ of all spinal injections [7]. Staphylococcus aureus is the most common causative organism. It is believed to be introduced via skin puncture, as a consequent of poor sterile technique. Undetected and untreated infection can lead to sepsis and spread to other sites through either direct contiguous spread or distant dissemination through the Batson's plexus [5].

Patients with meningitis typically present with headache, fever and nuchal rigidity. Kernig and Brudzinski signs may also be positive on physical examination. Cerebrospinal fluid cultures may demonstrate the causative organism and imaging may be normal early in the course of the disease. Hence, meningitis is a clinical/laboratory diagnosis, not an imaging diagnosis. Imaging is however important in delineating the possible complications of meningitis such as hydrocephalus, cerebritis, ventriculitis, abscess (Figures 10a and 10b), subdural empyema, dural venous sinus thrombosis and cerebral infarction secondary to vasospasm.

Enhancement of the pia mater that extends into the subarachnoid spaces of the sulci and cisterns is known as leptomeningeal enhancement and is usually associated with meningitis although similar findings may be seen in leptomeningeal metastases. The primary mechanism of this enhancement is breakdown of the blood-brain barrier. Glycoproteins produced by the infective organism result in breakdown of the bloodbrain barrier and allow contrast material to leak from vessels into the cerebrospinal fluid.

Abnormal enhancement of the ependymal surfaces of the ventricles may reflect concurrent ventriculitis (Figures 9a, 9b and 9c). Other features of ventriculitis include ventricular debris and periventricular FLAIR hyperintensities.

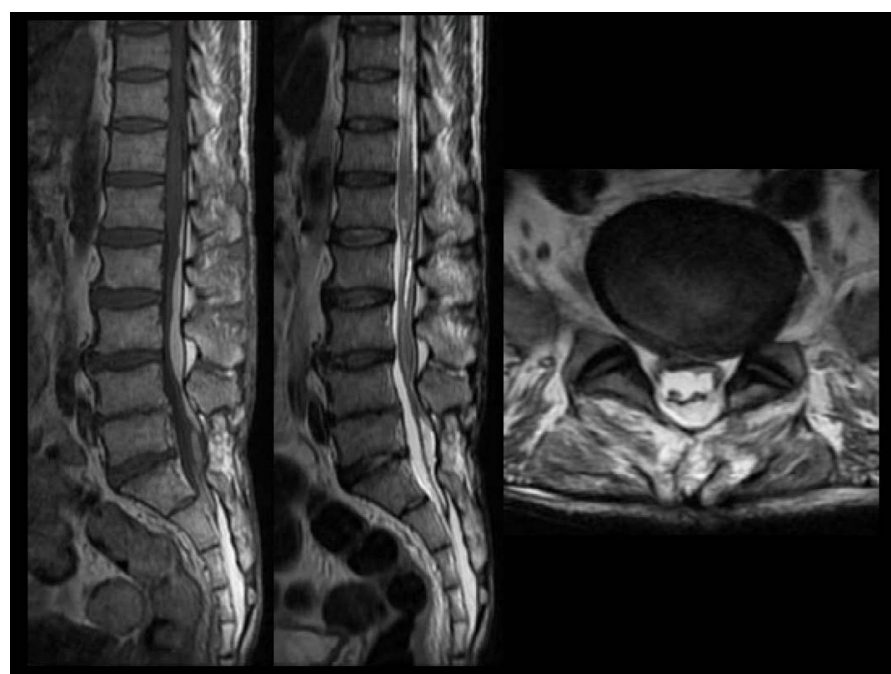

Figure 7. (a)Sagittal T1 and (b) T2-weighted images of the lumbosacral spine show a late subacute subdural hematoma extending from the inferior border of the T12 vertebra to the level of S2. (c)Axial T1-weighted image again shows the late subacute haematoma within the lumbosacral spinal canal

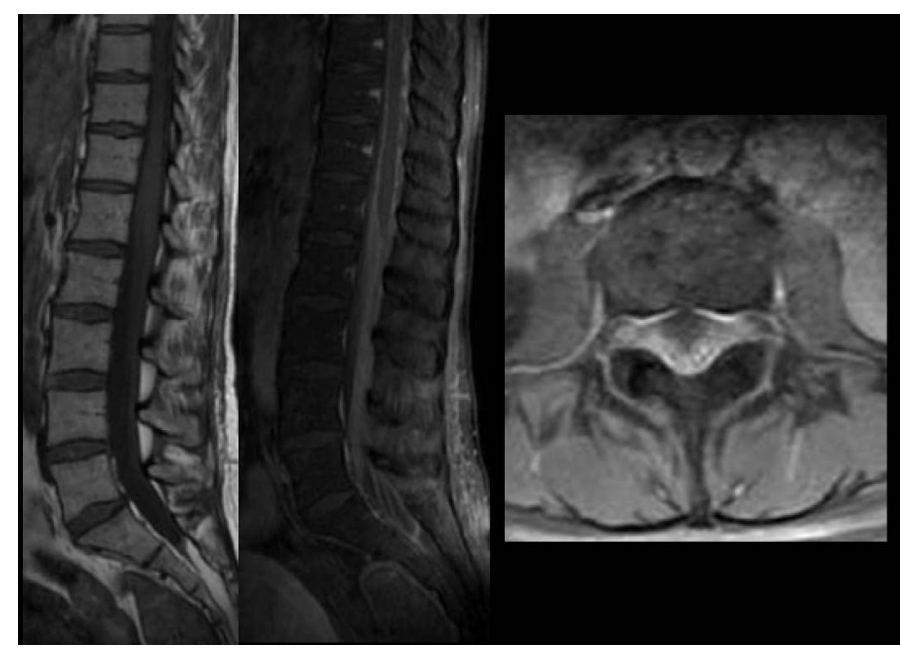

Figure 8. $(\mathrm{a}, \mathrm{b})$ Sagittal T1-weighted images pre- and post gadolinium administration demonstrate abnormal enhancement of the cauda equina nerve roots, in keeping with arachnoiditis (c)Axial T1-weighted image post gadolinium administration shows abnormal enhancement of the clumped descending and exiting nerve roots

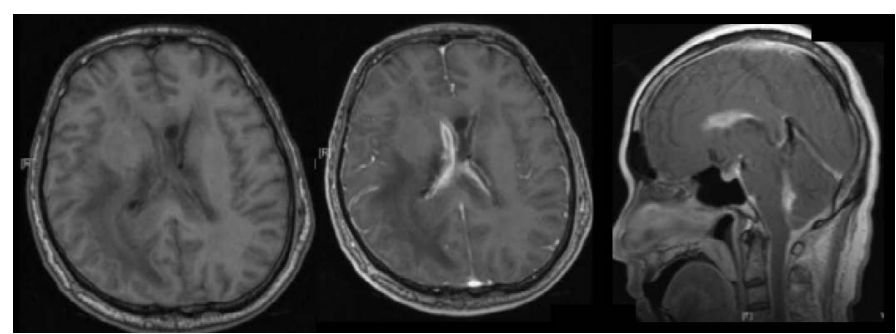

Figure 9. Axial T1-weighted images (a)pre- and (b)post gadolinium administration show abnormal enhancement of the ependymal surfaces in keeping with meningitis with concurrent ventriculitis. (c) Sagittal T1-weighted imaging post gadolinium administration of the same patient demonstrates abnormal ependymal enhancement within the lateral and fourth ventricles.

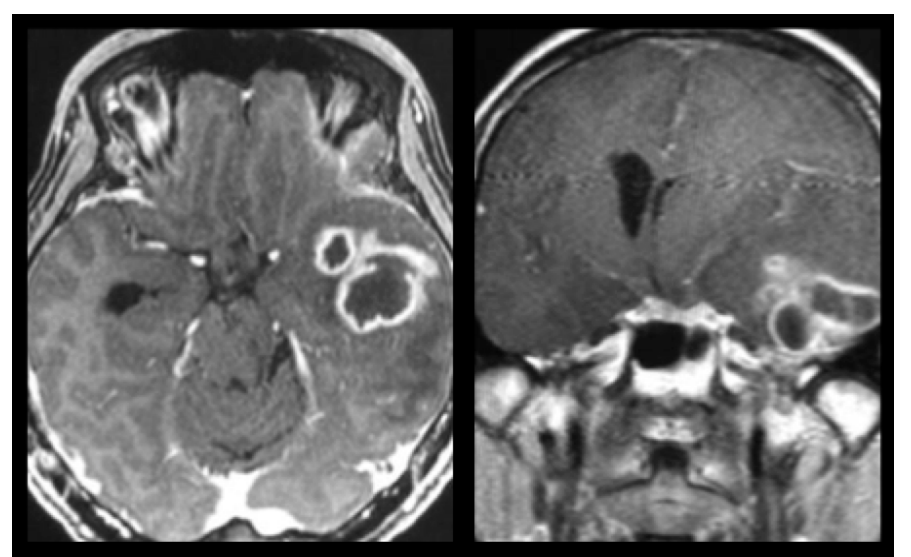

Figure 10. (a)Axial and (b)coronal T1-weighted images post gadolinium administration reveal rim enhancing abscesses in the left temporal lobe in a patient with concurrent meningitis (not shown in these images).

\section{Conclusion}

As the world heads into an era of dramatic demographic transformation where aging individuals encompass a larger proportion of the population, spinal degenerative disorders would be expected to be on the rise. Hence, it is very important that radiologists and clinicians are able to recognize complications of transforaminal epidural steroid injection (TFESI) on MR imaging. Familiarity with these complications would hasten diagnosis and improve patient's management. 


\section{Acknowledgement}

The authors wish to confirm that there are no known conflicts of interest associated with this publication and there has been no significant financial support for this work that could have influenced its outcome. We confirm that the manuscript has been read and approved by all named authors and that there are no other persons who satisfied the criteria for authorship but are not listed.

\section{References}

1. Tiso R, Cutler T, Catania J (2004) Adverse central nervous system sequelae after selective transforaminal block: the role of corticosteroids. Spine J 4: 468-474. [Crossref]

2. Lyders EM, Morris PP (2009) A case of spinal cord infarction following lumbar transforaminal epidural steroid injection: MR Imaging and Angiographic Findings. AJNR Am J Neuroradiol 30: 1691-1693. [Crossref]
3. Zhu J, Falco FJ, Formoso F, Onyewu O, Irwin FL (2011) Alternative Approach for Lumbar Transforaminal Epidural Steroid Injections. Pain Physician 14: 331-341. [Crossref]

4. Hoang JK, Apostol MA, Kranz PG, Kilani RK, Taylor JN, et al. (2010) FluoroscopyAssisted Cervical Transforaminal Steroid Injection: Tips, Traps, and Use of Contrast Material. AJR Am J Roentgenol 195: 888-894. [Crossref]

5. Botwin K, Gruber R, Bouchlas C (2000) Complications of fluoroscopically guided transforaminal lumbar epidural injections. Arch Phys Med Rehabil 81:1045-1050. [Crossref]

6. Stoll A, Sanchez M (2002) Epidural hematoma after epidural block: implications for its use in pain management. Surg Neurol 57: 235-240. [Crossref]

7. Windsor RE, Storm S, Sugar R (2003) Prevention and management of complications resulting from common spinal injections. Pain Physician 6: 473-483. [Crossref]

Copyright: $@ 2017$ Tan AP. This is an open-access article distributed under the terms of the Creative Commons Attribution License, which permits unrestricted use, distribution, and reproduction in any medium, provided the original author and source are credited. 